\title{
Prevalence and Risk Behaviors for Chlamydial Infection in a Population-Based Study of Female Adolescents in Brazil
}

\author{
ANGELICA ESPINOSA MIRANDA, MD, PHD, ${ }^{\ddagger}$ CELIA LANDMANN SZWARCWALD, PHD, $†$ RENATA LYRIO PERES, \\ AND KIMBERLY PAGE-SHAFER, PHD, MPH§
}

\begin{abstract}
Background: Adolescents are vulnerable to sexually transmitted infections (STIs) and unplanned pregnancy. Prevention measures and assistance are of significant public health importance in this population.

Objective: The objective of this study was to identify demographic, behavioral, and clinical factors for STIs and to determine the prevalence of Chlamydia trachomatis infection (CT) among female adolescents in Vitória, Brazil.

Methods: We performed a cross-sectional study among female adolescents (15-19 years) served by the Health Family Program. Participants were screened for CT and Neisseria gonorrhoeae (GC) using ligase chain reaction applied to urine and answered a face-to-face questionnaire to assess demographic, behavioral, and clinical factors. All participants and their parents signed the informed consent.

Results: Four hundred sixty-four young women were sampled. The prevalence of CT was $8.9 \%$ (95\% confidence interval [CI], 6.5-11.9\%) overall. Among sexually active women, $\mathrm{CT}$ and gonorrhea prevalence were 12.2 (95\% CI, 9.4-17.0\%) and $1.9 \%$ (95\% CI, 1.1-2.7\%), respectively. Previously diagnosed STI was reported by $12.8 \%$. Women who reported regular condom use and having condoms at home were significantly less likely to have CT, and having never purchased condoms was significantly associated with a positive CT result.

Conclusion: A high prevalence of CT was found in this population, and behavioral risk was high despite readily available STI prevention information. Women who reported positive condom use behaviors were less likely to have CT. These results demonstrate the need for ongoing STI prevention activities, including STI screening and continued successful risk reduction activities such as condom use to further decrease CT and other STI among adolescents.
\end{abstract}

ADOLESCENTS ARE AN ESPECIALLY important target group for primary sexually transmitted infection (STI) prevention because much of their active sexual and reproductive life lies ahead and they could be less inclined to appreciate their risk of acquiring STIs. ${ }^{1}$ However, the mere provision of information is usually not sufficient to allow patients to accurately assess their own risk of infection or to deal with the challenges of informing a partner/ partners, of preventing future infections, or dealing with complications of STIs. ${ }^{2}$

Funding for this study was provided by FACITEC (Fundo de Apoio à Ciência e Tecnologia), Vitória Municipality, UNESCO: Projeto 914 BRA $3016,5^{\circ}$ termo aditivo acordo Brasil-França and from the NIH/Fogarty Centers ICOHORTA Grant (1 D43 TW05799-03).

Correspondence: Angelica Espinosa Miranda, MD, PhD, Universidade Federal do Espírito Santo: CBM/NDI, Av. Marechal Campos, 1468 Vitória ES-29040090, Brazil. E-mail: espinosa@ndi.ufes.br

Received for publication December 22, 2003, and accepted March 29, 2004.

\begin{abstract}
From *Escola Nacional de Saúde Pública, †Centro de Informação Científica e Tecnológica, Departamento de Informações em Saúde, Fundação Oswaldo Cruz, Rio de Janeiro, Brazil; ‡Universidade Federal do Espírito Santo, Núcleo de Doenças Infecciosas, Vitoria, Brazil; and the §University of California, San Francisco, Center for AIDS Prevention Studies
\end{abstract}

Early sexual initiation places adolescents at risk of pregnancy and contracting STIs, including HIV. Sexual intercourse among adolescents is a common phenomenon in Latin America; it is estimated that $50 \%$ of adolescents under the age of 17 are sexually active. ${ }^{3}$ Little data exist about indicators of sexuality in adolescence in Latin countries or what constitutes sexually healthy adolescents within their cultural environment. ${ }^{4}$

Genital infections in women caused by Chlamydia trachomatis (CT) are associated with pelvic inflammatory disease, infertility, ectopic pregnancy, and chronic pelvic pain, and with conjunctivitis and pneumonia in newborns ${ }^{5-7}$ This infection is asymptomatic in most infected women 8 ; thus, efforts to prevent medical complications and to decrease transmission have focused on screening.

We assessed prevalence of CT in a population-based sample of female adolescents in the municipality of Vitória, Brazil, and identified demographic, behavioral, and clinical correlates of CT in this at-risk population. The information will be used for planning prevention and assistance programs to reach this population.

\section{Methods}

\section{Study Design}

This study was a single-stage, population-based, door-to-door, cross-sectional survey designed to measure the prevalence of CT and related risk behavior in adolescent women in Vitória, Brazil. Vitória is a port city in the state of Espírito Santo, located in the southeast region of Brazil. In collaboration with the Family Health Program (FHP), ligase chain reaction (LCx; Abbott Laboratories, Abbott Park, IL) was used to detect CT and Neisseria gonorrhoeae (GC) in urine specimens. The tests were performed at the laboratory of the Infectious Diseases Unit at the Universidade Federal do Espírito Santo (Federal University of Espírito Santo). A structured interviewer-administered survey was conducted to gather data on sociodemographic characteristics, sexual behavioral, substance use, medical history, and other health-related factors. Response rate for most variables was greater than $94 \%$. Parents were not present during the interview. 


\section{Recruitment}

An information campaign regarding the research project was conducted in collaboration with the FHP; information distributed in neighborhoods and group (parents and adolescents) and individual sessions (adolescents only) were held at the FHP offices. Information sessions for parents and adolescents included topics of reproductive health and its importance, confidentiality, some general health education sessions, and information about services provided by the FHP. Brazilian law forbids the use of monetary incentives for participation in research. The primary motivation for research participation is volunteerism and increased health knowledge. All adolescent participants were invited to attend a 4-session reproductive health education program at the FHP covering: hygiene, sexuality, contraception, and sexually transmitted diseases (STDs) and HIV.

\section{Study Subjects}

The target population was young women residing in Vitória, Brazil. Eligibility criteria were female sex, age 15 to 19 years, and residence in the target area. The Municipal Health Department of Vitória is divided administratively into 7 regions. Of these regions, Health Region II (Maruípe) was selected as the target area because the FHP had previously enumerated the entire population (a census), including adolescents, for targeted implementation of a wide variety of health services and programs aimed at lower-income families. Based on a priori power calculations, the study sought to enroll 464 women. The total population in this target area was 59,664 , of whom 3367 (5.6\%) were females ages 15 to 19. A random list was generated of dwellings with the target population (adolescent women); contact was made with a resident in 462 $(30.4 \%)$ dwellings, and $464(100 \%)$ young eligible women agreed to participate with active parental consent ( 2 homes had 2 eligible female participants). Recruitment occurred from March to June 2002. Urine samples were collected for 461 (99.4\%) of women who were interviewed. Women with positive CT and GC results received treatment according to Brazilian National Guidelines for treatment of STI, ${ }^{9}$ which was a single dose of $1 \mathrm{~g}$ azithromycin and/or $400 \mathrm{mg}$ ofloxacin orally.

\section{Statistical Methods}

Standard descriptive statistical analyses were performed, including frequency distributions for categorical data and calculation of means and standard deviations for continuous variables. Prevalence was calculated to reflect the relative frequency of each disease, with corresponding $95 \%$ confidence intervals (CIs). Bivariate and multivariate associations with $\mathrm{CT}$ were examined only among women who reported any history of sexual activity. Odds ratios and $95 \% \mathrm{CI}$ were calculated in bivariate analyses to estimate the strength of the association between CT infection and each potential risk factor. ${ }^{10}$ Independent risk factors for CT infections were assessed using stepwise logistic regression: variables significant at $P<0.05$ in bivariate analysis were entered into the model, and additional variables were entered into the multivariate model based on known a priori associations (for instance, age and number of sex partners). ${ }^{11}$ The National School of Public HealthFIOCRUZ ethics committee approved the protocol for the study. Written, informed consent was obtained by all participants and their parents according to Brazilian legal guidelines.

\section{Results}

All eligible female adolescents agreed to participate in the study. A total of 464 women participated; 3 refused testing for CT or GC.
Prevalence of CT was $8.9 \%$ (95\% CI, 6.5-11.9\%) overall $(\mathrm{n}=41)$. Sixty-nine percent $(\mathrm{N}=320)$ of the adolescents sampled reported any history of sexual activity of the sample, and prevalence of CT and GC was $12.2 \%$ (95\% CI, 10.4-14.0) and $1.9 \%$ (95\% CI, $1.1-2.7 \%)$, respectively. No CT or GC infections were found among women who reported no previous sexual activity. Compared with women who were sexually active, women who reported no history of sexual activity were significantly younger (median age 16.5 vs. 17.5 years, $P<0.001$ ), more likely to live with family (98.6\% vs., $70.3 \%, P<0.001)$, but did not report less schooling ( $70.8 \%$ vs. $62.5 \%$ reported some secondary education, $P=0.08$ ) or differences in history of family violence $(29.2 \%$ vs. $35.9 \%, P=$ $0.15)$.

Associations among sociodemographic, behavioral, and selected clinical history variables and CT among sexually active women are shown in Table 1. Among those women, median age was 17.5 years (interquartile range [IQR] 16-9), and median number of years of education was 9 (IQR 8-11), and median age at sexual debut was 15 years (IQR 14-16). The majority (65.9\%) reported being currently in school; and most $(89.7 \%)$ reported having access to STI and HIV prevention information. Two thirds (65\%) reported fear and concern regarding HIV infection. Having had an HIV test, reported by $25.9 \%$ of women, was significantly associated with a positive $\mathrm{CT}$ test (odds ratio [OR], 2.2; 95\% CI, $1.1-4.4 ; P<0.01)$. None of the women who reported no history of sexual activity had tested for HIV previously. A majority (76.6\%) of sexually active participants reported inconsistent (not always) condom use, and $24.4 \%$ reported having ever purchased condoms; both factors were significantly associated with CT infection, as was having more than 1 male sexual partner. Regular alcohol use, defined as "at least once per week," was reported by $68.8 \%$ of sexually active women, but this was not associated with CT infection. There was an association between drinking before/during sexual activity and do not use condoms (OR, 3.19; 95\% CI, $1.35-7.52 ; P=0.008)$. There was not statistical association when they reported that the partner had drunk before/during sexual activity. In addition to the high reported prevalence of familial violence (Table 1), almost one third of adolescent women $(28.9 \%)$ reported having experienced violence from a sexual partner.

Prevalence and associations of selected clinical and STI-related variables and CT infection among sexually active women are also shown in Table 1 . Less than half $(40.3 \%)$ of sexually active women reported having been examined by a gynecologist. Almost one third $(31.6 \%)$ of participants reported a previous pregnancy; however, this was not associated with CT. A history of previously diagnosed STI was reported by $12.8 \%$ of women. This variable and various STI symptoms, including vaginal discharge, pelvic pain, and itching, were significantly associated with CT (Table 1). Gonorrhea was only detected in a small proportion of participants $(1.9 \%)$; however, a positive test was significantly associated with CT.

Table 2 shows variables identified in multivariate analyses found to be independently associated with CT infection. Significantly, consistent (always) condom use and reporting having condoms at home were found to show significant protective associations with CT, and never having purchased condoms was associated with increased risk for number of lifetime sexual partners. Participant age was dropped from the model, because it had no independent association with CT, and dropping it had no effect on the risk estimates of the other variables in the model.

\section{Discussion}

This study of chlamydia infection in adolescent young women in Vitória, Brazil, showed a high prevalence of CT in sexually 
TABLE 1. Selected Socio-demographic, Behavioral, and Gynecological Risk Characteristics, and Associations With Chlamydia Infection Among Sexually Active Female Adolescents in Vitória, Brazil $(n=320)$

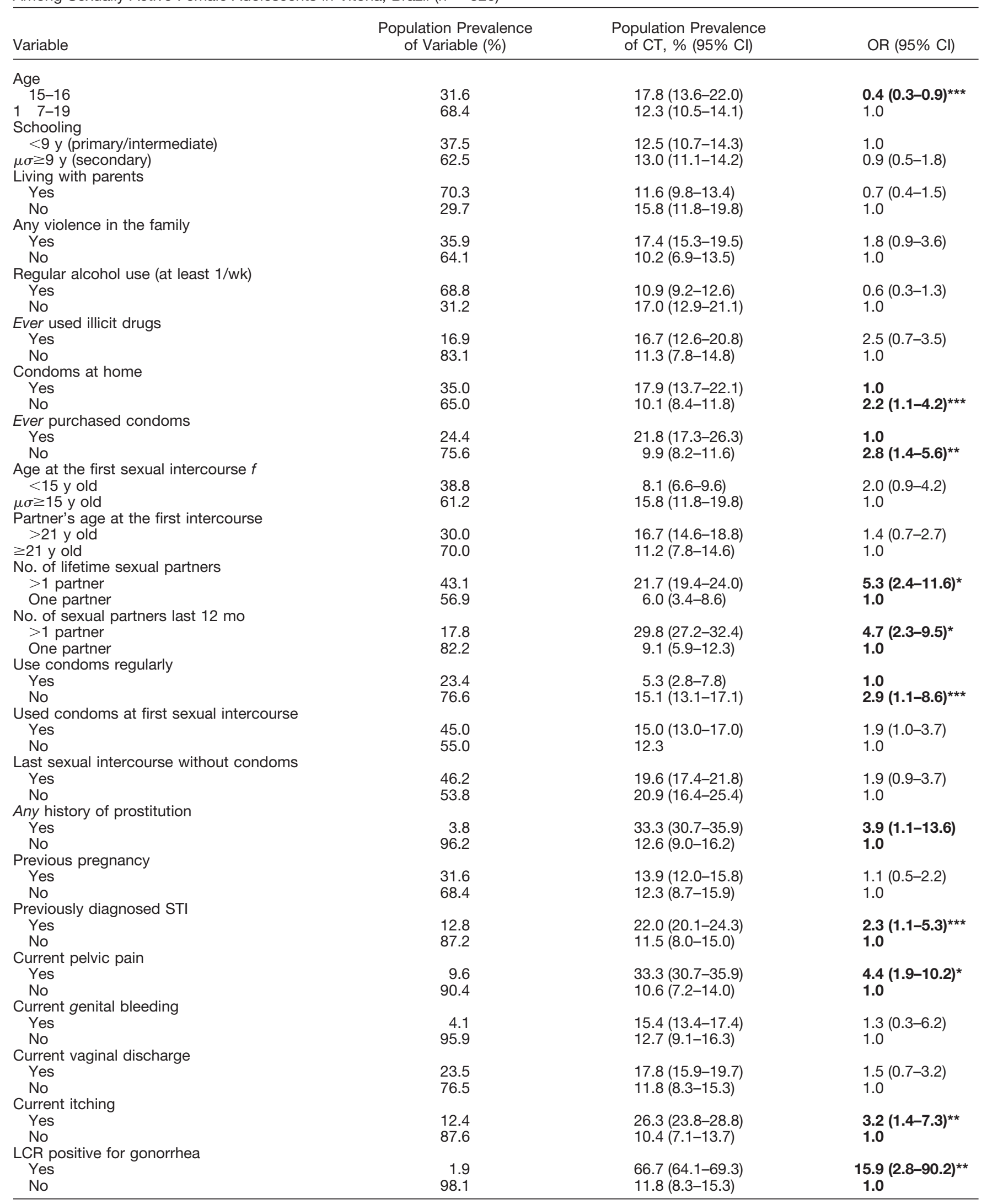

$\mathrm{Cl}=$ confidence interval; OR $=$ odds ratio; CT confirmed in $\mathrm{n}=39$.

${ }^{*} P<0.001,{ }^{* \star} P<0.01,{ }^{* \star *} P<0.05$.

$f$ : sexual intercourse question all refer to male sex partners. 
TABLE 2. Multivariate Analysis of Factors Independently Associated With Chlamydia Infection Among Female Adolescents in Vitória, ES, Brazil

\begin{tabular}{llll}
\hline Variables & OR & $95 \% \mathrm{Cl}$ & $P$ value \\
\hline More than one partner/life & 4.29 & $1.82-10.12$ & 0.001 \\
Have condoms at home & 0.46 & $0.21-1.00$ & 0.052 \\
Never bought condoms & 2.44 & $1.11-5.36$ & 0.027 \\
Regular condom use & 0.16 & $0.04-0.71$ & 0.016 \\
\hline
\end{tabular}

Overall percentage of total data used in analysis due to listwise deletion was 87.5; Hosmer and Lomeshow Test $=0.630(11)$.

active women $(12.2 \%)$. Because the population had been previously enumerated by the Family Health Program, this study sought to investigate $\mathrm{CT}$, gonorrhea, and risk correlates in a well-defined and representative population of women age 15 to 19 in Brazil. CT infection was significantly associated with number of sexual partners and significantly less likely among women who reported regular condom use.

The prevalence of CT infection observed in this study shows that CT infection rate is consistent with results of previous studies performed among older women in Brazil, which show rates ranging from $4.0 \%$ to $19.6 \% .12-21$ Our findings from this population based on a sample of young women in Vitória extend these earlier estimates and demonstrate that high rates of CT are not unique to the largest cities of Brazil nor older populations. These data are in agreement with data from North America, especially the United States where some studies show a high prevalence rate of CT among female adolescents as well high-risk behaviors. ${ }^{22-26}$ It shows that even in different cultures and socioeconomic societies, $\mathrm{CT}$ is very frequent.

The majority of participants report access to STI prevention information, yet high-risk behavior and self-reported STI symptoms were prevalent and associated with a positive CT result. These results are also consistent with findings among older Brazilian women. ${ }^{27}$ The high proportion of pregnancy and self-reported STI are especially worrisome. Countrywide, $18 \%$ of adolescents have been pregnant compared with $31.6 \%$ in this population-based sample. Although age of sexual debut (15 years) is lower in comparison to that in other surveys of Brazilian women, it is likely the result of the bias introduced by excluding sexually inactive women from the analyses.

In this study, the overall proportion of females tested for CT who had positive test results was high, indicating that there is an urgent need to implement the screening for CT. The ligase chain reaction test used in this study was noninvasive and well accepted by adolescent women. Special consideration must be given to the concerns of young women about vaginal examination that includes the use of a speculum and cervical swab. These data suggest that syndromic evaluation will not detect a large proportion of positive CT infections. The accuracy of the clinical diagnosis of genital infections is low, specifically as a result of the positive predictive value. In this study GC, pelvic pain and itching were strongly associated with CT. Although itching is not described as a symptom associated with $\mathrm{CT}$, it could be associated with a different and unmeasured STI. In comparison, GC was not highly prevalent, $(2 \%)$, similar to results from other studies. ${ }^{21}$

The significantly protective independent associations between factors related to condoms are especially notable. Young women who reported using condoms, having condoms at home, and having bought condoms were significantly less likely to have CT; Brazil has conducted widespread condom promotion campaigns countrywide for HIV prevention. These results suggest that the campaigns are reaching young women and that other infections, including perhaps HIV could be impacted.

Limitations of this study include the modest sample size, which could limit inference to other similarly aged women in Brazil. However, both the sampling methods and the high response rate are strengths that could outweigh this potential limitation. Despite having to obtain active parental consent, the participation rate in this study was excellent, most likely as a result of the strong information campaign conducted in collaboration with the FHP program targeted at both adolescents and parents. Significantly, the participation rate demonstrates that STI/HIV prevention programs can successfully implement confidential and private services for adolescents.

Continued and expanded STI prevention and services should be targeted to adolescent women. Young women in Brazil are at high risk for HIV and STI; STI symptoms, pregnancy rates, and low condom use suggest that risk perception is low among young women, and demonstrate the need for ongoing STI prevention activities, including STI screening and targeted risk reduction programs for adolescents. Increased educational efforts targeted at high-risk individuals are necessary to improve knowledge of STI and their adverse sequelae. With greater knowledge and awareness, individuals and couples could be more likely to practice protective behaviors such as reducing high-risk exposure and to increase preventive measures such as barrier contraception use.

\section{References}

1. Impact of HIV and Sexual Health Education on the Sexual Behaviour of Young People: A Review Update Report. Geneva: UNAIDS, 1997.

2. Walter HJ, Valghan RD. AIDS risk reduction among a multiethnic sample of urban high school students. JAMA 1993; 270:725-730.

3. The State of World Population 1997. New York: United Nations Population Fund, 1997.

4. Plan of Action for Health and Development of Adolescents and Youth in the Americas-1998-2001. Pan Am Health Organization, 1998.

5. Schachter J, Grossman M, Sweet RL, et al. Prospective study of perinatal transmission of Chlamydia trachomatis. JAMA 1986; 255 : $3374-3377$.

6. Cates WJ, Wasserheit JN. Genital chlamydia infections: Epidemiology and reproductive sequelae. Am J Obstet Gynecol 1991; 164:17711781.

7. Holmes KK, et al., eds. Sexually Transmitted Diseases, 3rd edition, New York: McGraw-Hill, 1999.

8. Wasserheit JN. Reproductive Tract Infections: Global Impact and Priorities for Women's Reproductive Health. New York: Plenum Press, 1998:61-91.

9. Brasil Ministério da Saúde. Secretaria de Projetos Especiais de Saúde. Coordenação de Doenças Sexualmente Transmissíveis e AIDS. Manual de Controle das Doenças Sexualmente Transmissíveis. Brasília, 1999.

10. Fleiss JL. Statistical Methods for Rates and Proportions, 2nd ed. New York: John Wiley \& Sons, 1981. 
11. Hosmer DW, Lemeshow S. Applied Logistic Regression. New York: John Wiley \& Sons, 1989.

12. Passos E, Focchi J, Cunha Filho JS, et al. Incidência de Chlamydia tracomatis e Neisseria gonorrhoea em mulheres assintomáticas nãopromíscuas e com doença inflamatória pélvica aguda. Rev Bras Ginecol Obstet 1995; 17:80-86

13. Amaral MG, Kulay L Jr, Granato C, et al. Infecção por Chlamydia tracomatis e fatores de risco em gestantes. Rev Ass Med Brasil 1995; 41:193-196.

14. Simões JA, Giraldo PC, Faúndes A. Prevalence of cervicovaginal infections during gestation and accuracy of clinical diagnosis. Infect Dis Obstet Gynecol 1998; 6:129-133.

15. Faúndes A, Telles E, Cristofoletti ML, et al. The risk of inadvertent intrauterine device insertion in women carriers of endocervical Chlamydia trachomatis. Contraception 1998; 58:105-109.

16. Melles HH, Colombo S, Linhares IM, Siqueira LFG. Avaliação de parāmetros para o diagnóstico laboratorial de infecção genital feminina pela Chlamydia trachomatis. Rev Soc Bras Med Tropical 2000; 33:355-361.

17. Varella RQ, Passos MRL, Pinheiro VMS, et al. Pesquisa de Chlamydia trachomatis em mulheres no Município de Piraí, Rio de Janeiro. J Bras Doenças Sex Transm 2000; 12(suppl):27-44.

18. Frias M, Passos MRL, Pinheiro VM, et al. Prevalência de infecção pela Chlamydia trachomatis em mulheres não grávidas. J Bras Doenças Sex Transm 2000; 13:5-22.

19. Bastos CA, Bravo RS, Lopes HR, et al. Identificação de Chlamydia trachomatis, Mycoplasma hominis e Ureaplasma urealyticum em gestantes e não gestantes. J Bras Doenças Sex Transm 2002; 14:31-37.

20. Araújo RSC, Guimarães BEM. Estudo da infecção genital por Chla- mydia trachomatis em adolescentes e jovens do sexo feminino no distrito sanitário Leste do Município de Goiānia: prevalência e fatores de risco. Rev Bras Ginecol Obstet 2002; 24:492.

21. Ramos MC, Becker D, Germany C, et al. Prevalência de Chlamydia trachomatis e Neisseria gonorrhoeae pela reação em cadeia da polimerase em urina de gestantes adolescentes e mulheres atendidas em ambulatórios de ginecologia em hospital público em Porto Alegre, Brasil. J Bras Doenças Sex Transm 2002; 14:4-8.

22. Marrazzo JM, White CL, Krekeler B, et al. Community-based urine screening for Chlamydia trachomatis with a ligase chain reaction assay. Ann Intern Med 1997;127:796-803.

23. Burstein GR, Waterfield G, Joffe A, Zenilman JM, Quinn TC, Gaydos CA. Screening for gonorrhea and chlamydia by DNA amplification in adolescents attending middle school health centers: Opportunity for early intervention. Sex Transm Dis 1998; 25:395-402.

24. Cohen DA, Nsuami M, Martin DH, Farley T. Repeated school-based screening for sexually transmitted diseases: A feasible strategy for reaching adolescents. Pediatrics 1999; 104:1281-1285.

25. Nsuami M, Cohen DA. Participation in a school-based sexually transmitted disease screening program. Sex Transm Dis 2000; 27:473479.

26. Wiesenfeld HC, Lowry DLB, Heine RP, et al. Self-collection of vaginal swabs for the detection of chlamydia, gonorrhea, and trichomoniasis: Opportunity to encourage sexually transmitted disease testing among adolescents. Sex Transm Dis 2001; 28:321-325.

27. Fernandes AMS, Antonio DG, Bahamondes LG, Cupertino CV. Conhecimento, atitudes e práticas de mulheres brasileiras, atendidas pela rede básica de saúde, com relação às doenças de transmissão sexual. Cadernos de Saúde Pública 2000; 16(suppl 1):103-112. 